\title{
Robustness analysis of digital image spearman's rho correlation
}

\author{
Wanghua Huang, Qinruo Wang, Weichao Xu and Yanzhou Zhou ${ }^{\mathrm{a}}$ \\ School of Automation, Guangdong University of Technology, Guangzhou, 510006, China
}

\begin{abstract}
The presence of salt-and-pepper noise in speckle image prevents the precise measurement of deformation and displacement fields in the traditional digital image correlation (DIC) technique. Spearman's rho (SR) has been introduced as a kernel correlation function in the DIC. The robustness of SR is quantitatively analyzed in following two cases: one is speckle images contaminated by same distribution salt-and-pepper noise, and the other is contaminated by random distribution noise. Theoretical and simulation results suggest that the DIC using SR is robust to about $10 \%$ salt-and-pepper noise. SR is suitable for measurement deformation and displacement fields.
\end{abstract}

Keywords: digital image correlation; salt-and-pepper noise; spearman's rho; deformation field distribution.

\section{Introduction}

The digital image correlation (DIC) technique, proposed by Yamaguchi[1] and Peters[2] in the 1980s, is often used for measurement of deformation and displacement fields. It is a non-contact, full-field, and high precision measurement technique.

During the acquisition and transmission of a digital image, various types of noise occur from the effects of the shooting environment and/or image sensor. For example, thermal noise is caused by the thermal agitation of the charge carriers; fixed-pattern noise is caused by the difference between photosensitive diodes; salt-and-pepper noise is caused by a strong interference or failure of analog-to-digital converters[3], etc. Pan proposed Gaussian pre-filtering[4] and Savitzky-Golay filter[5]. Sutton used a combination of a generalized cross-validation and a finite element algorithm[6]. Sun determined the size using subset entropy[7]. Yao proposed a wavelet analysis technique for denoising in DIC[8].

Salt-and-pepper noise is a common type of noise in digital images[9, 10]. Even a single this kind of noise will lead to serious error in traditional DIC applications. The Spearman's rho correlation[11-13], proposed as another DIC criterion[14], is robust to salt-and-pepper noise. In this manuscript, we focus on its robustness in DIC.

a Corresponding author : zhouyanzhou@gdut.edu.cn 


\section{Theory}

\subsection{Spearman's rho correlation}

Let $\left\{\left(A_{i}, B_{i}\right)\right\} n i=1$ denote $n$ independent and identically distributed (i.i.d.) data pairs drawn from a bivariate population with continuous joint distribution. Let $P_{i}$ be the rank of $A_{i}$ and $Q_{i}$ be the rank of $B_{i}$, the SR correlation coefficient $C_{S R}$ is defined as [15]:

$$
C_{S R}(A, B)=1-\frac{6 \sum_{i=1}^{n}\left(P_{i}-Q_{i}\right)^{2}}{n\left(n^{2}-1\right)} \text {. }
$$

A pair of reference and target speckle images is regarded as a bivariate Gaussian model. The parent of $\{(A, B)\}$ (i.i.d.) is a bivariate Gaussian distribution, whose density function is

$$
\phi(a, b)=\frac{1}{2 \pi \sigma_{A} \sigma_{B} \sqrt{1-\rho^{2}}} \exp \left[-\frac{z}{2\left(1-\rho^{2}\right)}\right],
$$

where

$$
z=\frac{\left(a-\mu_{A}\right)^{2}}{\sigma_{A}^{2}}-\frac{2 \rho\left(a-\mu_{A}\right)\left(b-\mu_{B}\right)}{\sigma_{A} \sigma_{B}}+\frac{\left(b-\mu_{B}\right)^{2}}{\sigma_{B}^{2}},
$$

$\rho$ is the correlation coefficient between $A$ and $B ; \mu_{A}$ and $\mu_{B}$ are the gray means, $\sigma 2 A$ and $\sigma 2 B$ are the gray variances of image $A$ and $B$ respectively.

The above bivariate Gaussian model can be expressed as:

$$
(A, B) \sim N\left(\mu_{A}, \mu_{B}, \sigma_{A}^{2}, \sigma_{B}^{2}, \rho\right) .
$$

When the two speckle images are contaminated by salt-and-pepper noise with a huge variance, the following contaminated Gaussian model can represent the probability density function of the two contaminated speckle images[11]:

$$
C N\left(\rho, \rho^{\prime}\right)=(1-\varepsilon) \mathrm{N}\left(\mu_{A}, \mu_{B}, \sigma_{A}^{2}, \sigma_{B}^{2}, \rho\right)+\varepsilon \mathrm{N}\left(\mu_{A}, \mu_{B}, \lambda_{A}^{2} \sigma_{A}^{2}, \lambda_{B}^{2} \sigma_{B}^{2}, \rho^{\prime}\right),
$$

where $0 \leq \varepsilon \leq 1, \lambda_{A} \gg 1, \lambda_{B} \gg>1,-1 \leq \rho \leq 1$, and $-1 \leq \rho^{\prime} \leq 1 . \rho$ is the population correlation coefficient of the two "clear" speckle images, and $\rho^{\prime}$ is the population correlation coefficient of the salt-and-pepper noise in the two speckle images.

\subsection{Robustness analysis to salt-and-pepper noise}

When $\varepsilon$ is sufficiently small, the approximate mean of the SR correlation coefficient can be defined as[12]:

$$
\lim _{\substack{n \rightarrow \infty \\ \lambda_{A} \rightarrow \infty \\ \lambda_{B} \rightarrow \infty}} E\left(\mathrm{C}_{S R}\right)=\frac{6}{\pi}\left[(1-3 \varepsilon) \sin ^{-1} \frac{\rho}{2}+\varepsilon \sin ^{-1} \rho^{\prime}\right] .
$$

The approximate mean of $C_{S R}$ between the reference contaminated speckle image and the target contaminated speckle image is composed of $\rho$ and $\rho^{\prime}$, as $n \rightarrow \infty, \lambda_{A} \rightarrow \infty$, and $\lambda_{B} \rightarrow \infty$.

To guarantee the two contaminated speckle images are still highly correlated, we propose that the correlation coefficient should be mainly decided by $\rho$ in DIC. 


$$
\left\{\begin{array}{c}
(1-3 \varepsilon) \sin ^{-1} \frac{\rho}{2}>0 \\
(1-3 \varepsilon) \sin ^{-1} \frac{\rho}{2}>\varepsilon \sin ^{-1} \rho^{\prime}
\end{array} .\right.
$$

In an extreme case where the two speckle images are contaminated by same distribution salt-and-pepper noise $\rho^{\prime}=1$, then

$$
\left\{\begin{array}{c}
0 \leq \varepsilon<\frac{1}{3} \\
(1-3 \varepsilon) \sin ^{-1} \frac{\rho}{2}>\frac{\pi \varepsilon}{2}
\end{array}\right.
$$

We have $0 \leq \varepsilon \leq 0.1467$. That is, the SR correlation coefficient between the reference and the target speckle image is mainly decided by $\rho$, when $0 \leq \varepsilon \leq 0.1467, \rho^{\prime}=1, \rho \geq 0.8$, and $n \rightarrow \infty$.

Similarly, when the two speckle images are contaminated by random distribution salt-and-pepper noise, assuming $\rho^{\prime}=0.5$, we can obtain $0 \leq \varepsilon \leq 0.2341$.

\section{Simulation}

\subsection{Simulation schemes}

There are two simulation groups conducted. The first one involves testing for $\rho^{\prime}=1$, when the two speckle images are contaminated by same distribution salt-and-pepper noise. In the other, the two speckle images are contaminated by same density but random distribution salt-and-pepper noise $\left(\rho^{\prime} \neq 1\right)$. Figure 1 shows the reference and target speckle images, contaminated by $6 \%$ random distribution salt-and-pepper noise, and the simulated displacement vector field.
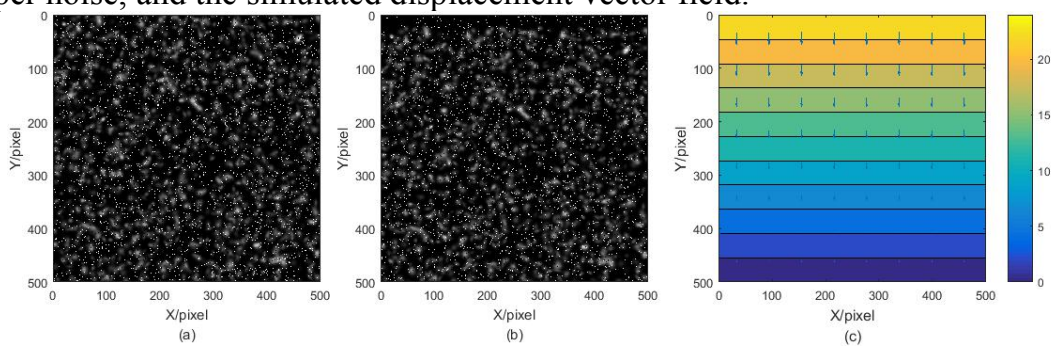

Figure 1. Speckle images contaminated by $6 \%$ random distribution salt-and-pepper noise. (a) Reference speckle image; (b) target speckle image compressed on Y axis (ratio:0.95); (c) simulated displacement vector field.

\subsection{Simulation result and analysis}

\subsubsection{Contaminated by same distribution salt-and-pepper noise}

Figure 2(a) shows the mean of the EVM(Error Vector Magnitude) using SR when the reference and the target speckle images are contaminated by same density and same distribution salt-and-pepper noise $(2 \%-40 \%)$ for various sub-block sizes $(31 \times 31$ to $61 \times 61)$. The population correlation of salt-and-pepper noise $\rho$ is 1 . The Figure 2(b-e) are the displacement vector fields.

As shown in Figure 2(a), the mean of the EVM is mainly decided by the noise density, and is almost consistent for different sub-block sizes with the exception of the $31 \times 31$ sub-block. It maintains less than two pixels when the density is less than $12 \%$. The mean of the EVM shows a rapid increase 
as the density moves from $14 \%$ to $28 \%$. It stays at approximately 12 pixels when the density is greater than $28 \%$, regardless of the size of the sub-block.

From Equations (6-8) in Section 2, if the density is less than $14.67 \%$, the result is decided by the "clear" speckle images, when $\rho^{\prime}=1$, and the size of the sub-block is infinite. However, the size of sub-block is a finite value, in fact. As shown in Figure 2(a), the mean of EVM is about one pixel when the density is less $10 \%$ and the size of the sub-block is larger than $35 \times 35$. The effects of the displacement vector fields are very good in Figure 2(b) and Figure 2(c), when the noise is less than $10 \%$ and the sub-block size is $51 \times 51$.
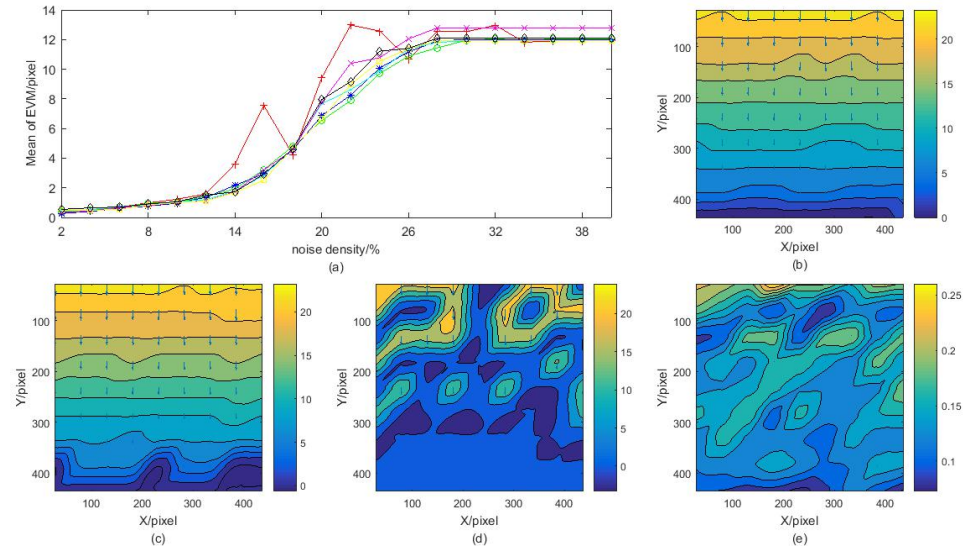

Figure 2. Result when contaminated by same distribution salt-and-pepper noise.(a) EVM mean, Plus: $31 \times 31$; circle: $35 \times 35$; asterisk: $41 \times 41$; point: $45 \times 45$; cross: $51 \times 51$; square: $55 \times 55$; diamond: $61 \times 61$, (b-e) Calculated displacement vector fields: (b) $6 \%$ noise, (c) $10 \%$ noise, (d) $20 \%$ noise,(e) $30 \%$ noise.

If the density is between $14 \%$ and $28 \%$, the result is decided by the "clear" speckle images and the salt-and-pepper noise. The mean gradually increase as shown in Figure 2(a), but the displacement vector fields is disorderly, as in Figure 2(d).

Moreover, if the density is greater than $33.33 \%$, the result will be decided by the salt-and-pepper noise, as in the right part of Figure 2(a). When the density is larger than $32 \%$, the result will maintain the same value, because $\rho^{\prime}$, the population correlation coefficient of the salt-and-pepper noise in the two speckle images is 1 . All the displacement vectors will point to themselves, as shown in Figure 2(e).

\subsubsection{Contaminated by random distribution salt-and-pepper noise}

Figure 3 show the results of the second simulation group using SR. Figure 3(a) shows the mean of the SR correlation coefficient, which monotonically decreases as the noise density increases. Figure 3(b) shows the mean of the EVM when speckle images are contaminated by same density $(2 \%-32 \%)$ but random distribution noise for various sub-block sizes $(31 \times 31$ to $61 \times 61)$. The sub-graph represents the results for $31 \times 31$ and $35 \times 35$ sub-block sizes.

As shown in Equation (6) and Figure 3(a), the population correlation of "clear" speckle images is about 0.98 , ignoring the effect of $2 \%$ noise. We can also easily find that the population correlation $\rho^{\prime}$ of random distribution salt-and-pepper noise is approximately $0.45-0.5$. To make sure the result is decided by the "clear" speckle images, the tolerable density of SR is less than $23.41 \%$ in theory, if the size of sub-block is infinite. 

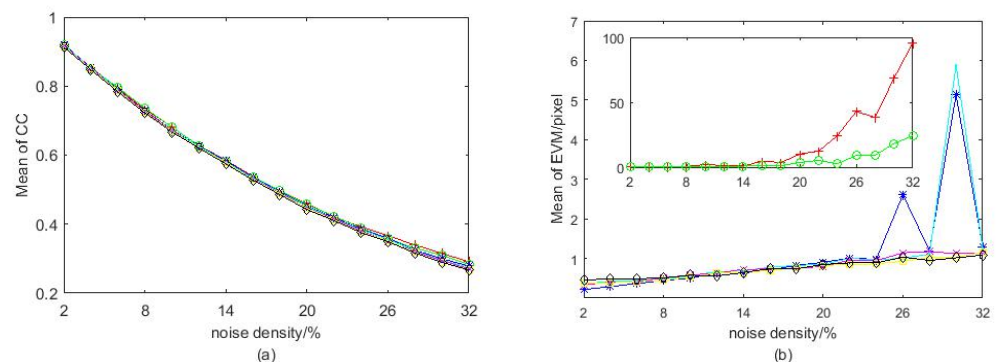

Figure 3. Simulation result of random distribution salt-and-pepper noise. (a) mean of SR correlation coefficient; (b) EVM mean. Plus: $31 \times 31$; circle: $35 \times 35$; asterisk: $41 \times 41$; point: $45 \times 45$; cross: $51 \times 51$; square: $55 \times 55$; diamond: $61 \times 61$.

The mean of the EVM is larger when the sub-block size is $31 \times 31$ or $35 \times 35$, sometimes up to approximately 100 pixels in Figure 3(b). There are two peak values when the size is $41 \times 41$, and one peak value at $45 \times 45$. It is more stable when the sub-block size is $51 \times 51$, particularly when the sub-block size is $55 \times 55$ or $61 \times 61$. The mean of the EVM is less than one pixel when the density is less than $20 \%$ and the size is larger than $51 \times 51$.

\section{Conclusion}

Analyzing the mean characteristic of SR used in DIC shows that, the SR can do well with less than $14.67 \%$ same distribution salt-and-pepper noise or less than $23.41 \%$ random distribution noise. Because SR is based on the rank, it is little affected by spike noise. Less than $10 \%$ noise will be better for high accuracy and avoiding extreme cases. Based on the adaptable noise density, a sub-block size larger than $51 \times 51$ is recommended in this manuscript (speckle radius: $3-6$ pixels).

\section{Acknowledgement}

Work partially supported by the National Natural Science Foundation of China under project 11072063; in part by the Guangdong Natural Science Foundation under project 2014A030313519; and in part by the Guangzhou Municipal Science and Technology Project 2014J4100203.

\section{References}

1. I. Yamaguchi. A laser-speckle strain gauge. Journal of Physics E: Scientific Instruments, 1981, 14(11): 1270-1273.

2. W.H. Peters, W.F. Ranson. Digital Imaging Techniques In Experimental Stress Analysis. Optical Engineering, 1982, 21(3): 213427-213427.

3. C.Q. Davis, D.M. Freeman. Statistics of subpixel registration algorithms based on spatiotemporal gradients or block matching. Optical Engineering, 1998, 37(4): 1290-1298.

4. B. Pan. Bias error reduction of digital image correlation using Gaussian pre-filtering. Optics and Lasers in Engineering, 2013, 51(10): 1161-1167.

5. B. Pan, H. Xie, Z. Guo, et al. Full-field strain measurement using a two-dimensional Savitzky-Golay digital differentiator in digital image correlation. Optical Engineering, 2007, 46(3): 033601-033601-10.

6. M.A. Sutton, J.L. Turner, H.A. Bruck, et al. Full-field representation of discretely sampled surface deformation for displacement and strain analysis. Experimental Mechanics, 1991, 31(2): 168-177.

7. Y. Sun, J.H.L. Pang. Study of optimal subset size in digital image correlation of speckle pattern images. Optics and Lasers in Engineering, 2007, 45(9): 967-974. 
8. X. Yao, Z. Wu, G. Jin. Wavelet denoising using the digital speckle correlation method. Journal Tsinghua University, 2001, 41(4/5): 108-111.

9. C.-T. Lu, T.-C. Chou. Denoising of salt-and-pepper noise corrupted image using modified directional-weighted-median filter. Pattern Recognition Letters, 2012, 33(10): 1287-1295.

10. R.H. Chan, H. Chung-Wa, M. Nikolova. Salt-and-pepper noise removal by median-type noise detectors and detail-preserving regularization. Image Processing, IEEE Transactions on, 2005, 14(10): 1479-1485.

11. G.L. Shevlyakov, N.O. Vilchevski. Robustness in Data Analysis: criteria and methods2002: Walter de Gruyter.

12. W. Xu, Y. Hou, Y.S. Hung, et al. A comparative analysis of Spearman's rho and Kendall's tau in normal and contaminated normal models. Signal Processing, 2013, 93(1): 261-276.

13. R. Ma, W. Xu, Q. Wang, et al. Robustness analysis of three classical correlation coefficients under contaminated Gaussian Model. Signal Processing, 2014, 104(0): 51-58.

14. W. Huang, Q. Wang, W. Xu, et al. Robust digital imaging Spearman's rho correlation for pepper and salt noise. Optics and Precision Engineering, 2015, 23(6): 1800-1806.

15. J.D. Gibbons, S. Chakraborti. Nonparametric Statistical Inference(3rd)1992, New York: M Dekker. 